\title{
Theorizing space-time relations in education: The concept of chronotope
}

\author{
Giuseppe Ritella ${ }^{\text {1a }}$, Maria Beatrice Ligorio ${ }^{\mathrm{b}}$ \& Kai Hakkarainen ${ }^{\mathrm{a}}$ \\ ${ }^{a}$ University of Helsinki, Finland \\ ${ }^{\mathrm{b}}$ University of Bari, Italy
}

Article received 15 September / revised 21 December / accepted 21 December / available online 19 January

\begin{abstract}
Due to ongoing cultural-historical transformations, the space-time of learning is radically changing, and theoretical conceptualizations are needed to investigate how such evolving space-time frames can function as a ground for learning. In this article, we argue that the concept of chronotope - from Greek chronos and topos, meaning time and place/space lends itself well to reach this aim. In particular, we outline three features of chronotope: 1) its analytical focus includes the examination of the potential interdependency between space and time; 2) it allows us to examine space and time as social constructions, negotiated in dialogical interaction; 3) it involves the analysis of both the material organization and the discursive negotiation of space and time. We use examples from our own studies and from relevant literature to illustrate how these features of the concept allow us to examine the role that space-time relations play in educational practice. Finally, we draw our conclusions and briefly introduce the theoretical and methodological challenges to be addressed for a full development of the concept.
\end{abstract}

Keywords: space-time, ecological perspectives on learning, co-construction of knowledge, agency, materiality, trans-contextuality, chronotope 


\section{Introduction}

This article is framed as a theoretical contribution to a special number of the journal whose purpose is to discuss how existing conceptualizations of educational practices should be redefined to address emerging learning issues. The aim of the article is twofold: 1) to argue for the relevance of analysing spacetime relations as an emerging issue in research on learning, and 2) to propose chronotope as a useful theoretical concept, allowing us to uncover the time-space relations in local investigation sites.

The rationale of our argumentation is that we live in an historical moment in which both technological innovations and educational reforms are triggering deep changes in space-time relations in learning. The introduction of continuously evolving virtual spaces and the implementation of pedagogical approaches such as the flipped classroom (Flipped Learning Network, 2014), connected learning (Ito et al., 2010) and place-based learning (van Eijck \& Roth, 2010) entail the transformation of the spatial and temporal organization of learning. For example, in a school using the flipped learning approach, lessons can be shared through internet-based software and studied at home, while in the classroom the students can engage in collaborative learning. The time-space organization of learning and teaching change, based on the pedagogical use of technology. Historically, such transformations of space-time have been crucial in changing educational practices:

"The organization of a serial space was one of the great technical mutations of elementary education. It made it possible to supersede the traditional system (a pupil working for a few minutes with the master, while the rest of the heterogeneous group remained idle and unattended). By assigning individual places it made possible the supervision of each individual and the simultaneous work of all. It organized a new economy of the time of apprenticeship. It made the educational space function like a learning machine, but also as a machine for supervising, hierarchizing, rewarding." (Foucault, 1977, p. 155)

Accordingly, in order to understand what current transformations imply for learning, we need conceptual and analytical tools that allow examining the space-time relations that emerge in the empirical sites of investigation. We claim that the concept of chronotope can be productively used to reach this aim. Crafted from the ancient Greek words chronos and topos, meaning time and place/space, chronotope was devised by Mikhail Bakhtin (1981) both to examine the space-time patterns characterising literary genres and to develop a framework for the cultural analysis of space-time (Holquist, 1982). Recently, educational research has shown increasing interest in this concept. Following Bakhtin, this literature is based on the assumption that space and time are interdependent social constructions rather than independent given realities (van Eijck \& Roth, 2010).

A literature review of the uses of the concept is beyond the scope of this short article. Instead, by drawing on our own studies and on some of the relevant literature, we discuss how chronotope can be adopted for enriching our understanding of learning in the twenty-first century. First, we will briefly introduce the main features of chronotope as a conceptual tool for the analysis of space-time frames. Second, we will refer to three significant socio-cultural studies that have addressed issues related to space-time relations. We have selected these three studies because we think that they are valuable contributions to sociocultural research; they are also relevant for developing a theory of space-time in education. In sum, we seek to demonstrate that we can gain further insight about learning processes by carrying out a specific analysis of the (discursive) social negotiation and bodily-material organization of space-time. Finally, theoretical and methodological challenges will be discussed. 


\section{The concept of chronotope}

Three features of chronotope make its use fruitful for examining space-time relations:

a) its analytical focus includes the examination of the potential interdependency between space and time;

b) it allows us to examine space and time as social constructions, negotiated in dialogical interaction;

c) it involves the analysis of both the material organization and the discursive negotiation of space and time.

In the following paragraphs, we will briefly discuss each feature of chronotope, illustrating through examples how it contributes to make salient situated time/space arrangements.

First, space and time are interdependent when considered for the analysis of human action, making it crucial to examine space and time in a coordinated way. A clear example of this interdependence can be found in the different space-time organization of a literature review carried out by means of a search engine such as Google Books and the same review carried out in a physical library. The absence of a digital search engine implies that one has to take the time to visit the library, consult the physical book records, often organised alphabetically by author names, titles or fields/topics of the books. An alternative is to ask for help from the library staff. All of that must be done before going to find the actual physical books on the shelves and then finding the relevant text within each book. These actions should be carried out in this order, unless one decides to engage in a (most likely ineffective) exploration of the shelves, opening and skimming systematically those books having interesting titles. In contrast, some digital search engines enable a search for books in any location connected with the internet through a full-text search of one or more keywords and obtaining immediate access to the pages where the searched keyword appears. From that point, a researcher has the chance to form an idea of the contents of interest, and then decide whether to read the full text and if so, how carefully. Analysing these two cases by using chronotope implies to examine spatial relations (e.g. location of events, spatial arrangements of workspaces, etc.) and temporal relations (e.g. duration, temporally ordered sequences of actions, rate of recurrence of events, etc.) in a coordinated way. Thus, it allows recognizing that the introduction of a virtual space containing a digital search engine in the workspace involves a transformation of the entire temporal structure and duration of the activity. The other way around, temporal limitations can affect the selection of tools and the organization of the spaces of the activity. For example, in one of our investigations (Ligorio \& Ritella, 2010), we used the concept of chronotope to analyse how a group of teachers changed their bodily positions around the computer throughout a session of collaborative problem solving. We identified different spatial arrangements of bodies and technology and different tempos of the activity. The analysis showed that one particular spatial arrangement, where all the teachers were standing around the same computer, was realized in order to speed up the accomplishment of the task when the end of the session was approaching.

In observing that space and time are interdependent, we do not mean that all transformations of space involve a transformation of time and vice versa. It is possible that some changes in space are neutral with regard to the organization of time and vice versa. For example, changing the spatial organization of the objects on a desk does not necessarily implies changes in the temporal organization of the activity. However, theoretically it is important to acknowledge the existence of such interdependence, as described in the two examples above. It is a goal for researchers to determine under which conditions such interdependence is relevant for educational practice.

Second, the concept of chronotope was introduced by Bakhtin as a concept for the cultural analysis of space and time (Holquist, 1981). This implies considering all the different voices involved in social processes, in contrast to the "philosophical monopolization" of scientific discourse, which considers space and time as given realities external to human experience (van Eijck \& Roth, 2010). Therefore, the task set by using the concept of chronotope is not just to measure physical spaces and time intervals according to the 
consolidated scientific paradigm. The scientific understanding of space-time is just one of the possible voices to be considered for understanding space-time relations, and the other voices - for instance, the voices of the participants - should not be silenced in the analysis. Thus, there is a need to consider dialogue in the analysis of space-time. In other words, the concept of chronotope is devised to examine space and time as they are socially negotiated in dialogue. Indeed, they are not simply 'given' to the participants involved in an activity: meanings associated with both space and time are socially negotiated, and the organization of activities in space-time can be highly flexible. Van Eijk and Roth (2013), for example, examined an environmental education project in which students from aboriginal Canadian communities engaged in nature conservation practices in a marine park. By using the lens of chronotope, the authors managed to understand how the aboriginal students and the collaborators to the project constructed the material space of the marine park in dialogical interaction. In particular, they illustrated how science education activities carried out during the project involved conflicting notions of space and time derived from the perspective of natural science and from the local culture. Analysing these tensions allowed the researchers 1) to identify some contradictions in the project and 2) to trace how the students experienced the science education activities while developing their cultural identities as Aboriginals.

Third, as stated by many authors (Hirst \& Vadeboncouer, 2006; van Eijck \& Roth, 2010; Matusov, 2009), chronotope concerns both the immaterial, semiotic, worlds of discourse and narratives, and "patterns of organization of space and time" (Lemke, 2004) that are enacted through the movement of bodies and objects. Therefore, chronotopic analysis takes into account also the bodily-material aspect of space-time. Given such ground, many scholars have used chronotope to investigate space-time at the boundary between material and discursive processes. For example, Brown and Reshaw (2006) discuss how students express their agency by actively shaping the space-time contexts of the classroom, drawing on past, present and future temporal relations though discursive interaction. In particular, they analysed: 1) how a student initially built her private space-time within the classroom by using a library shelf and a desk, and in a second moment removed those barriers to actively enter into the shared space-time of collaborative activity; 2) how the students discursively shifted between different space-times while explaining and justifying their ideas and developing their identities. Another good example is the study by Hist and Vadeboncoeur (2006). Here the authors analyse how the re-engagement in schooling by a dropout student was mediated by the construction of a dynamic spatial network, which involved movements between material spaces (a learning centre and the student's home) and the concomitant reframing of the student-teacher relationship. In both these studies, the use of the concept of chronotope allowed to uncover how space-time arrangements affected the learning processes.

In the present article, chronotopes are defined as "socially emergent" (Sawyer, 2005) units of spacetime, where both discursive and material aspects of space-time relations are considered. In line with the literature, human cognition and learning are not conceived as located within the boundaries of the mind, but are distributed in the space-time context of the activity. Contexts - including space-time - emerge from a continuous process of social negotiation engaged in by learners (Bateson, 1972; Cole, 1996; Duranti \& Goodwin, 1992). During learning activities, participants individually or jointly attend to various physical and symbolic spaces: they organise their workspace, co-ordinate their efforts, and perceive space-time constraints and opportunities related to the technological tools used and to the institutional regulation of space and time. Following Bakhtin, we consider these spatial and temporal processes to be fused, requiring a co-ordinated analysis. Examining only space or only time could bias our understanding, given the reciprocal impact they can have on each other. 


\section{Sketching a conceptualization of chronotope for 21st-century learning}

As argued above, chronotope is a concept specifically intended for the analysis of space-time relations. Given the features of chronotope that we have presented above, the goal we set for chronotopic analysis is to investigate: 1) how patterns of space-time organization are involved in different learning activities, different schools systems, different pedagogical approaches; 2) how participants make sense of space-time patterns in dialogical interaction; 3) how participants' discursive negotiation of space-time is related to their bodily-material organization of space and time. This topic is not new to education. Space and time are ubiquitous categories of human experience, and this is reflected in the research literature. Indeed, most studies in the learning sciences involve references to space and/or time co-ordinates, although it is rare to find studies that specifically address space and time, or better put, space-time, as the primary focus of investigation. Below, we will discuss how specific attention to space-time attained through the concept of chronotope can enrich and extend the knowledge derived from three investigations in which the categories of space and time were relevant.

First, Silseth and Arnseth $(2011 ; 2015)$ provide an interesting example by examining learning across sites from a dialogical perspective. The authors analyse how ideas and perspectives that emerge in out-ofschool situations, and external representations produced in the past, are mobilized in situated interaction. These resources contribute to creating connections between different situations of learning. Space and time are highly relevant for this topic of investigation, which concerns learning processes taking place in multiple locations across extended periods of time. However, Silseth and Arnseth seem to consider space and time as a background against which the learning takes place, failing to consider them as analytical foci. We argue that the role played by the organization of space-time in this process can be examined by using the concept of chronotope. For example, in a previous case study (Ritella \& Ligorio, 2016) we studied the collaborative sensemaking of a group of professionals working on the design of a web-platform. We discussed how the space-time organization of the activities that took place before face-to-face meetings of the group is connected with the emergence of ideas and viewpoints during subsequent meetings. We found that providing an online space for writing individual notes foregrounded the emergence of the personal perspectives of the participants during the upcoming meeting. In contrast, arranging a physical meeting resulted in the emergence of a collective perspective by a subgroup of participants. Thus, we argued that chronotope helped to uncover how the space-time organization of activities affects sensemaking across multiple locations and extended periods of time.

Second, Engle (2006) shows that the way teachers discursively frame the context of learning, including the definition of temporal boundaries, affects students' transfer of knowledge across different contexts. In particular, the author analyses how a teacher framed learning episodes as building on previous ones or as relevant for the future. The continuous references to the past and the future helped the students to consider these episodes as interconnected, thus supporting the transfer of knowledge across contexts. The author states that space is also relevant for analysing the framing of context, and further research is needed to understand its role. The concept of chronotope, we argue, could be employed to direct analytical attention to both space and time relations, and analyse them in a co-ordinated way, adding further insights. Ryan (2011), for example, used the concept of chronotope to examine how students discursively conceive the space-time of university. This study shows that space and time jointly contribute to define the students' orientation towards academic life. In particular, some students depicted the university as a site of mass education with large lecture theatres, no permanent space for student groups and limited time for individual meetings with teachers because of the busy life of the academic staff. Put together, time limits and spatial arrangements of university buildings generated a conception of the university as a potentially distant service provider, which encouraged students to spend most of their time off-campus. Thus, using the concept of chronotope allowed detecting how the discursive construction of space-time can have an effect on the students' academic practices. 
A last example is the research by Jornet and Roth (2015), who discussed how students make sense of multiple material representations of scientific phenomena across time. In particular, the authors trace the students' bodily and pragmatic actions in interaction with representations of scientific phenomena, and discuss how these relate to the students' experiences and interpretations. We recognise that Jornet and Roth significantly discuss such a relationship by means of their analysis. However, we claim that this topic of investigation requires also a specific analysis of space-time relations that was missing in their study. The material representations used by the students are distributed in space and they are picked up at different times. Conceptualizing space-time relations as chronotopes might allow researchers to uncover how the students spatially arrange their bodies around, and attend to, multiple representations at different times. In a previous study (Ritella, Ligorio \& Hakkarainen, 2015), we have used the concept of chronotope to examine how a group of teachers managed the resources available in the context during a session of collaborative Problem Solving (PS). We interpreted, diachronically, the alternation of 1) events in which the participants explored the space and actively searched for resources in the environment, and 2) events characterized by a focus on a stable set of resources. One of the findings of this study was that in some phases of PS the first type of events was dominant. These moments were associated with: (a) the introduction of a new task, (b) the use of a software suite not yet mastered by the teachers or (c) a change in the configuration of participation (i.e. the participants physically changed their positions in the room, or changed the set of tools used) realised in conjunction with a difficulty in trying to solve the problem. We expect that patterns of this kind could be found also while analysing the students' use of multiple representations. For example, it could be found that material representations play a crucial role in some phases of educational activities and/or that the way in which they are spatially organized affects their usage by the students. Thus, a better understanding of when the students explore the space around them and when they attend to each representation could give us further insights on how they interact with the material environment during learning practices.

In sum, in this section we have shown that a specific focus on space-time can yield additional insights for the analysis of learning. For instance, we could see how learning may be affected by the psychical organization of the space within which students interact and how the perception of time constrains influences how the task is perceived. As we will further discuss in the next section, the concept of chronotope has great potentials for examining learning practices as they unfold in space and time, even though some challenges have still to be tackled for the full deployment of the concept.

\section{Implications and challenges for chronotopic analysis}

In this paper, we argue that the concept of chronotope can enrich the (dialogical) understanding of learning practice. We have briefly presented this concept and discussed how it could be used to enhance and extend the findings of investigations that implicitly or explicitly address space and time relations in learning. We define chronotope as the emergent configuration of temporal and spatial relations in educational practices. To provide some examples of how chronotopes can be fruitfully used to analyse learning practices, we have discussed how the discursive/bodily/material organization of space-time is connected to:

a) The learning processes taking place at multiple locations across extended periods of time, as theorised by Silseth and Arnseth (2011);

b) The participants' discursive framing of situations of learning, as outlined by Engle (2006);

c) The use of multiple representations during a learning activity, as examined by Jornet and Roth (2010).

We believe that the range of applications of chronotope extends far beyond the processes we have discussed here. Surely, considering how space-time is organised by participants may be useful in designing learning tasks, especially when technology is involved. Indeed, contemporary digital environments offer multiple types of resources; (re)organising them in time and space during an activity is not a trivial task. A 
complex orchestration is needed to use all resources effectively. The same applies in designing training situations for teachers. The appropriation of technology from the teachers' side is not only a matter of understanding the technical features. Developing awareness of how the time-space is transformed by technology may help teachers in improving their educational practices. The changes that technology introduces go beyond the local classroom situation. As in a cascade effect, these changes ultimately cause modification in the larger society. Renshaw (2014) pointed out that the school systems can be characterised by different chronotopes in different historical periods.

We are aware that the conceptualization sketched in this paper is not yet fully developed and has some limitations. One of them is that current methodological tools are not yet adequate to grasp the organization of space-time in all its complexity. Indeed, the organization of space and time applies to different units of analysis. Both micro-processes, such as the situational co-ordination of a group of students, and macro-level processes, such as the historical development of school systems, involve patterns of organization of space and time. This suggests that the operationalization of chronotope and the methods used should be adapted to the unit of analysis in each investigation. Moreover, another challenge is that the negotiation of space and time can often be implicit and difficult to detect. In one of our studies (Ritella, Ligorio \& Hakkarainen, accepted), while negotiating the meaning of a task set by teachers, the students broadly discussed the negotiation of time, but the discussion about space was marginal during the observed interaction. One possible interpretation was that space had been taken for granted and did not emerge clearly in the students' discourse. However, this could be attributed also to a methodological limitation. The students may have engaged in discussions about space during breaks or informal meetings, when the researcher did not observe the interactions. Therefore, a more comprehensive research design should be planned, able to make explicit the conceptions of space and time while preserving the privacy of the participants.

Based on the discussion here presented, we argue that continuing to pursue this frontline concept is important for advancing our understanding of contemporary learning practices because space-time relations have undergone profound transformations. Acknowledging the ongoing transformations of space and time in education involves theoretical and methodological challenges to research. We believe that the concept of chronotope, thanks to its focus on space and time as interconnected social constructions, which are discursively negotiated and bodily-materially enacted by participants, lends itself well to addressing these challenges.

\section{Aknowledgments}

The authors are grateful to Feldia Loperfido and Antti Rajala for their comments on a previous version and to the editors and reviewers for constructive criticism that have allowed the development of a stronger article.

\section{References}

Bakhtin, M. (1981). The dialogic imagination. Four essays by M. M. Bakhtin. Austin: University of Texas Press.

Bateson, G. (1972). Steps to an ecology of mind: Collected essays in anthropology, psychiatry, evolution, and epistemology. Chicago: The Chicago University Press.

Brown, R., \& Renshaw, P. (2006). Positioning students as actors and authors: A chronotopic analysis of collaborative learning activities. Mind, Culture and Activity, 13(3), 244-256. doi: 10.1207/s15327884mca1303_6

Cole, M. (1996). Cultural Psychology: A once and future discipline. Cambridge: Harvard University Press. 
Duranti, A., \& Goodwin, C. (Eds.). (1992). Rethinking context: Language as an interactive phenomenon. Cambridge: Cambridge University Press.

van Eijck, M., \& Roth, W. M. (2010). Towards a chronotopic theory of "place" in place-based education. Cultural Studies of Science Education, 5(4), 869-898. Doi: 10.1007/s11422-010-9278-2

van Eijck, M., \& Roth, W. M. (2013). Place and Chronotope. In Imagination of Science in Education (pp. 133-162). Springer Netherlands.

Engle, R. A. (2006). Framing interactions to foster generative learning: A situative explanation of transfer in a community of learners classroom. The Journal of the Learning Sciences, 15(4), 451-498. doi: $10.1207 / \mathrm{s} 15327809 \mathrm{jl} 1 \mathrm{~s} 1504 \_2$

Flipped Learning Network (FLN). (2014) The Four Pillars of F-L-I-PTM

Foucault, M. (1977) Discipline and punish: the birth of the prison. Vintage.

Hirst, E., \& Vadeboncoeur, J. A. (2006). Patrolling the borders of otherness: Dis/placed identity positions for teachers and students in schooled spaces. Mind, Culture, and Activity, 13(3), 205-227. doi: $10.1207 / \mathrm{s} 15327884 \mathrm{mca} 1303 \_4$

Holquist, M. (1982). Bakhtin and Rabelais: Theory as praxis. Boundary 2, 5-19.

Ito, M., Gutiérrez, K., Livingstone, S., Penuel, B., Rhodes, J., Salen, K., \& Watkins, S. C. (2013). Connected learning: An agenda for research and design. Irvine, CA: Digital Media and Learning Research Hub.

Jornet, A., \& Roth, W. M. (2015). The joint work of connecting multiple (re) presentations in science classrooms. Science Education, 99(2), 378-403. Doi: 10.1002/sce.21150

Lemke, J. (2004). Learning across multiple places and their chronotopes. In AERA 2004 Symposium, April (pp. 12-16).

Ligorio, M. B., \& Ritella, G. (2010). The collaborative construction of chronotopes during computersupported collaborative professional tasks. International Journal of Computer-Supported Collaborative Learning, 5(4), 433-452. Doi: 10.1007/s11412-010-9094-4

Matusov, E. (2009). Journey into dialogic pedagogy. Nova Science Publishers.

Renshaw, Peter D. (2013). Classroom chronotopes privileged by contemporary educational policy: teaching and learning in testing times. In Phillipson, S., Kelly Y. L. Ku and Shane N. Phillipson (Ed.), Constructing Educational Achievement: A sociocultural perspective (pp. 57-69). Oxon, UK: Routledge.

Ritella, G., Ligorio, M. B., \& Hakkarainen, K. (2015). The role of context in a collaborative problem-solving task during professional development. Technology, Pedagogy and Education, 25(3), 395-412. Doi: 10.1080/1475939X.2015.1062412

Ritella, G., \& Ligorio, M. B. (2016). Investigating chronotopes to advance a dialogical theory of collaborative sensemaking. Culture \& Psychology, 22(2), 216-231. doi: 10.1177/1354067X15621475

Ritella, G., Ligorio, M. B., \& Hakkarainen, K. (accepted) Interconnections between the discursive negotiation of space-time and the interpretation of a collaborative task. Learning, Culture and Social Interaction.

Ryan, M. (2011). Productions of space: Civic participation of young people at university. British Educational Research Journal, 37(6), 1015-1031. Doi: 10.1080/01411926.2010.517827

Sawyer, R. K. (2005). Emergence: Societies as complex systems. Cambridge: Cambridge University Press.

Silseth, K., \& Arnseth, H. C. (2011). Learning and identity construction across sites: A dialogical approach to analysing the construction of learning selves.Culture \& Psychology, 17(1), 65-80. Doi: $10.1177 / 1354067 \mathrm{X} 10388842$

Silseth, K., \& Arnseth, H. C. (2015). Frames for learning science: analyzing learner positioning in a technology-enhanced science project. Learning, Media and Technology, 1-20. Doi: 10.1080/17439884.2015.1100636 